\title{
Nanoparticles of Palladium, Platinum and Silver: Incoporation into Perfluoro-Sulfonated Membrane MF-4SK and Ionic Nafion
}

\author{
Alexandra Revina*, Sergey Busev, Anatoly Kalinitchev, Michail Kuznetsov, \\ Ardalion Ponomarev, Marina Lebedeva \\ Institute of Physical Chemistry and Electrochemistry, RAS, Moscow, Russia \\ Email: *Alex_revina@mail.ru
}

Received December 28, 2012; revised February 2, 2013; accepted February 10, 2013

Copyright (C) 2013 Alexandra Revina et al. This is an open access article distributed under the Creative Commons Attribution License, which permits unrestricted use, distribution, and reproduction in any medium, provided the original work is properly cited.

\begin{abstract}
The purpose of the investigation is the study of the physico-chemical properties and electro-catalytic characteristics of the Nafion and MF-4SK membranes with the author's nanoparticles (A. Revina, 2008) incorporated into the perfluorosulphonated cationic membranes. An important advance in the creation of new nano-composite materials with polyfunctional activity is the inclusion of nanoparticles of various metals $(P d, P t, A g)$ in these membranes. Polymer ion exchange membranes represent widely applicable materials in various areas of modern nanotechnologies. The obtained nanocomposites on the base of included nanoparticles have the perspective properties and polyfunctional activity for the applications.
\end{abstract}

Keywords: UV-VIS Spectrophotometry; AFM; CVA-Measurements; Palladium Nanoparticles; Platinum Nanoparticles; Silver Nanoparticles; Perfluoro-Sulfonated Membranes; Nafion; MF-4SK Membrane

\section{Introduction}

Proton conductive sulpho-cationite type membranes "Nafion" (USA, Dupont Co) together with the Russian analogies (MF-4SK membranes) are often used for fuel cells operating at ambient temperature, and besides in addition for the electrochemical investigations of catalysis.

In recent years, while solar energy, geothermal energy, wind energy and fusion power technology have attracted much attention there is also the increasing interest for the very efficient hydrogen utilization in the generation of electrical energy. Among the different competing fuel technologies, polymer electrolyte cells have the most attractive advantages. The development of "Nafion" (Nf) membranes by DuPont and the exact structure of the perfluorinated ionomers have been intensively investigated. However, the morphology of $\mathrm{Nf}$ and its unique properties have not yet been clearly identified [1]. The article by Sahu et al. [2] presents an overview of $\mathrm{Nf}$ membranes highlighting their merits and demerits with an emphasis on the modified Nf membranes. Hydrated Nf clusters may be used as a reactive vessel (or template) for other

${ }^{*}$ Corresponding author. materials such as metals. Yan-Li et al. [3] reported that Pt NanoParticles (NPs) without the carbon support could be prepared by an alcohol method by using an anionic $P t$ complex. In particular, $P t$ NPs are of great interest because of their excellent catalytic activity. Their catalytic activity depends on the size distribution and morphology of the particles and, therefore, synthesis of the stable Pt NPs with the possibility of the size control could be determinative for these applications in membranes intended for the fuel cells.

Nafion is produced by the co-polymerization of variable amounts of unsaturated perfluoro-alkylsulfonylfluoride with tetrafluoro-ethylene. The chemical structure of the Nf is depicted at Figure 1. The quantitative characteristic: Equivalent weight $(\mathrm{Ew})$ is calculated by the grams of the dry $\mathrm{Nf}$ per mole of sulfonic acid groups with the assumption that the material is in the acid form.

These membranes and the analogous Russian MF-4SK membranes [4] are widely used in Polymer Electrolyte Fuel Cells (PEFCs) due to their high proton conductivity and moderate swelling in water. Nafion has clusters (about $\sim 40 \AA$ ) of sulfonate-ended perfluoralkyl ether groups organized as reverse (inverted) micelles arranged 


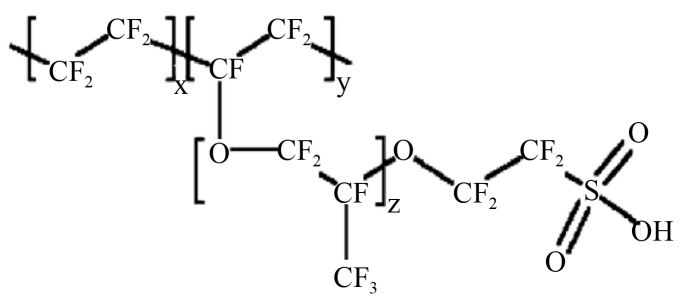

Figure 1. Chemical structure of Nafion (Nf).

on a lattice, (Figure 2(a)). These micelles are connected by pores or channels with $10 \AA$ in size. The channels with $-\mathrm{SO}_{3}^{-}$groups invoke the inter-cluster ion hopping of the positively charged species (Figure 2(b)).

Proton transport in the $\mathrm{Nf}$ membranes has been studied and widely reported in the literature. It depends on the water content of the membrane although the precise mechanism for the proton transfer in the solvated form of $\mathrm{Nf}$ is not completely understood. Generally, it is assumed that the state of water in the membrane is not fixed. Some part of the water is tightly bound to $-\mathrm{SO}_{3}^{-}$groups and is called "chemically bound water", which has a lesser degree of hydrogen bonding than bulk water. The latter is described as "physically bound water" presenting in the central pore region of the Nafion membrane or in the water core of reverse micelles [5]. Proton conductivity in the $\mathrm{Nf}$ occurs through the ionic channels formed by micro- or nano-phase separation between the hydrophilic proton exchange sites and the hydrophobic domains.

The schematic presentation of the reverse micelle (Figure 3(a)) and chemical formula for the surface active substance (SAS)-AOT (bis(2ethylhexyl)sulfosuccinate sodium salt) are depicted in Figure 3(b). The reverse micelles are often used as nano-reactors in the synthesis of nano-sized structures, which are stable in both: the liquid phase and in the adsorbed state [6,7].

As can be seen in Figures 2 and Figure 3(a), the states of the water inside the $\mathrm{Nf}$ pores and in the water pool of the reverse micelles are similar. The layers with $-\mathrm{SO}_{3}^{-}$groups in the reverse micelles invoke the inner water pool with positively charge species: as metal ions and $\mathrm{H}_{2} \mathrm{O}^{+}$. The physical chemistry of reverse micellar systems has attracted attention in nano-science and nanotechnology. Much consideration has been given to the physical chemistry of reverse micellar systems in recent years.

The micro-structural characteristics of water/AOT/isooctane micro-emulsions have been investigated by NMR spectroscopy [8]. It was observed that the aqueous core of the micelle is composed of bound and free water, while a small amount of the water remains trapped in the interface. The water structure in the aqueous core of reverse micelles exhibits behavior close to the biological membranes or protein interface, which is markedly different from that of the bulk water.

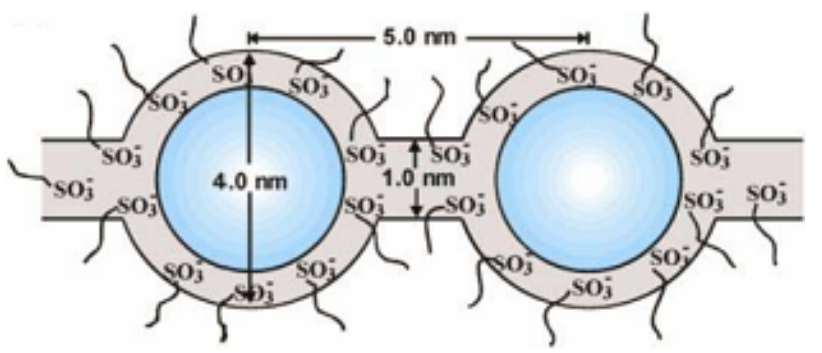

(a)

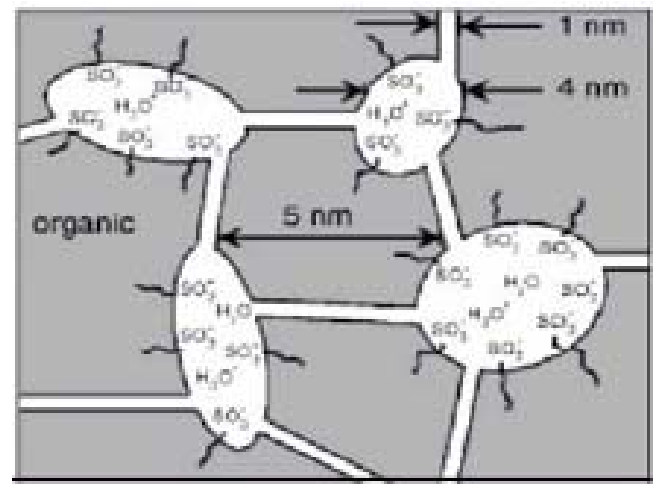

(b)

Figures 2. Cluster-network model for the interaction between polymer and water in Nafion membrane [2].

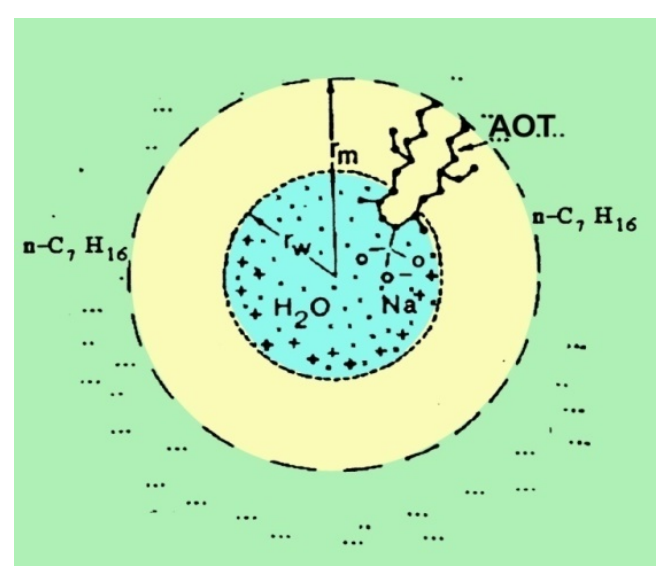

(a)

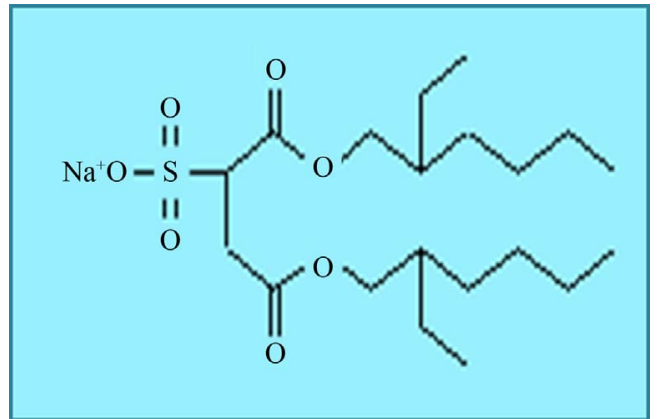

(b)

Figure 3. (a) Schematic diagram of reverse micelles; (b) Chemical structure of the surface active substance AOT (bis(2ethylhexyl)sulfosuccinate sodium salt). 
On the basis of the volt-ampere data the conclusion was made, that the electro-conductivity of the reverse micellar system does not depend on the applied voltage and is reduced by electrolysis. It was shown by PMR that the water proton chemical shift depends both on the water and electrolyte concentration in the reverse micelles water pools. In addition, it is changed by electrolysis. The hypothesis was suggested that the mechanism of the RMS electric conductivity is represented by an electronhole approach where the scattering of lattice defects is defined by the force of the hydrogen bonds in the micelle pool [9].

\section{Experimental}

\subsection{Materials}

In this study, we report a similar synthetic route for $A g$, $P d$ and $P t$ NPs with different shapes and sizes by using reverse micelles as nano-reactors for the formation of NPs and control of their concentration due to adsorption because of the contact with the polymer perfluoro-sulfonated cationic MF-4SK membrane and ionic Nf. Stable $P d$, (or $A g$ ) NPs in the liquid phase were obtained by the radiation-chemical reduction of $\mathrm{Pd}^{2+}$ ( or $\mathrm{Ag}^{+}$) ions in the reverse micellar system: $\mathrm{H}_{2} \mathrm{O} / \mathrm{AOT}\left(0.15 \mathrm{~mol} \cdot \mathrm{L}^{-1}\right) /$ isooctane with different solubilization coefficients- $\omega$ according to the technique described earlier [6,7]. In all experiments below the solubilization coefficient $(\omega)$ is determined by the relation: $\omega=\left[\mathrm{H}_{2} \mathrm{O}\right] /[\mathrm{AOT}]$.

\subsection{Apparatus}

The optical adsorption spectra (OAS) of reverse micellar solutions, containing metallic NPs were recorded in the $190-800 \mathrm{~nm}$ range with a quartz cell $(l=1 \mathrm{~mm})$ using a

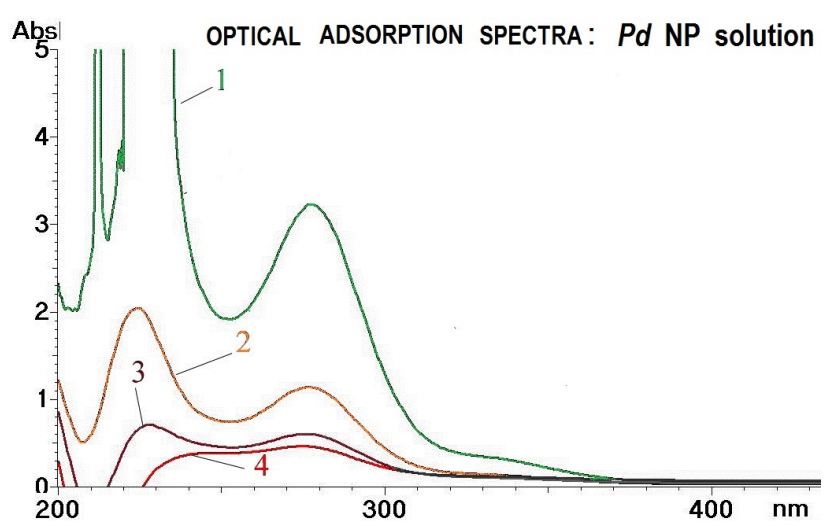

(a) $P d$ NPs
"Hitachi" U-3310 spectrophotometer (Japan). Analyses of the NPs adsorption spectra were completed for the initial micellar solutions and for the same solution after contact with the samples of the polymer membrane MF4SK (or Nf) or by interaction of the NPs with MF-4SK (or Nf) in the micellar solutions for different times. It was established by atomic force microscopy (AFM) that the size of NPs (Z-size) from the micellar solutions was in the range of $1-10 \mathrm{~nm}$ depending on $\omega_{0}$ with the doses (5 - $15 \mathrm{kGy)}$ of $\gamma$-irradiation carried out with a RKhM $\gamma$ 20 setup.

The electro catalytic activity and the electrode stability were measured by cyclic voltammetry (CVA) with using of the device IPC-PRO M (Technopribor, Russia).

There are described the experimental preparations of the metal-ion exchanger nanocomposites by adsorption of the $P d$ NPs, $P t$ NPs and $A g$ NPs dispersed in the inversed micelle solution on the surface, and in the pores of the perfluor-sulfonated cationic MF-4SK or Nf membranes. The properties of the obtained nanocomposites and metal NPs in the membranes and in the Nf films are analyzed by the various methods: OAS, AFM and CVA. The obtained results are presented below.

\section{Results and Discussion}

The adsorption for the metal nanoparticles and the introduction of these nanoparticles inside the polymer matrix were observed via optical adsorption spectra (OAS, Figures 4 and 5 ).

The OAS of the $P d$ nanoparticles (NPs) in the micellar solution with $\omega=5.0$ are shown on Figure 4(a) (1-initial spectrum). The micellar solutions of the $P d$ NPs (with $\omega=5.0$ ) have two adsorption maxima at $225 \mathrm{~nm}$ and $280 \mathrm{~nm}$ (Figure 4(a), curves 1-3).

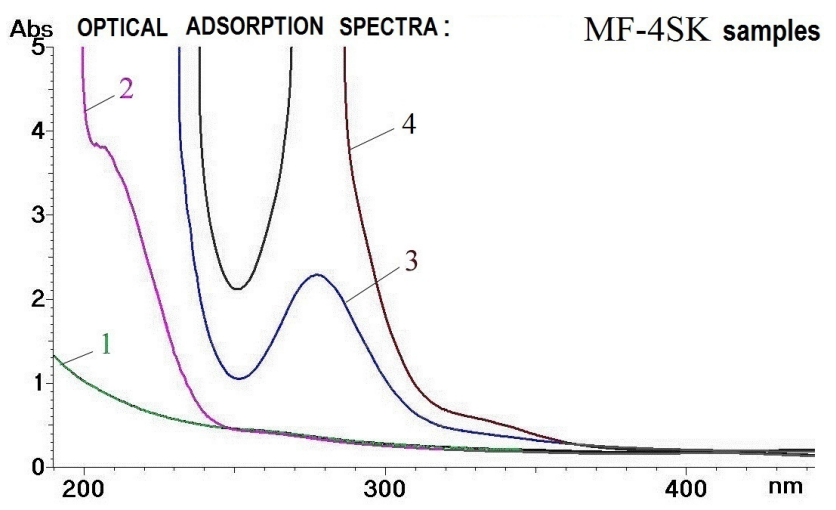

(b) $P d \mathrm{NPs}+$ membrane samples

Figure 4. (a) Evolution of OAS (190 - $500 \mathrm{~nm})$ for $P d$ NP solution $(\omega=5.0)$ after contact with MF-4SK membrane: $1-$ initial moment (0 min); 2-after $30 \mathrm{~min}$; 3-after $60 \mathrm{~min}$; 4-after $120 \mathrm{~min}$ and (b) OAS (190 - $500 \mathrm{~nm}$ ) for different MF-4SK membrane samples: 1 -initial membrane; 2 -membrane after 2 days, contact with AOT/isooctane solution (without Pd NP); 3 membrane after 2 days contact with solution $P d$ NP $(\omega=3.0) ; 4-$ membrane after 2 days contact with solution $P d$ NP $(\omega=$ 5.0). $\mathbf{t}^{0}=20^{\circ} \mathrm{C}$. 


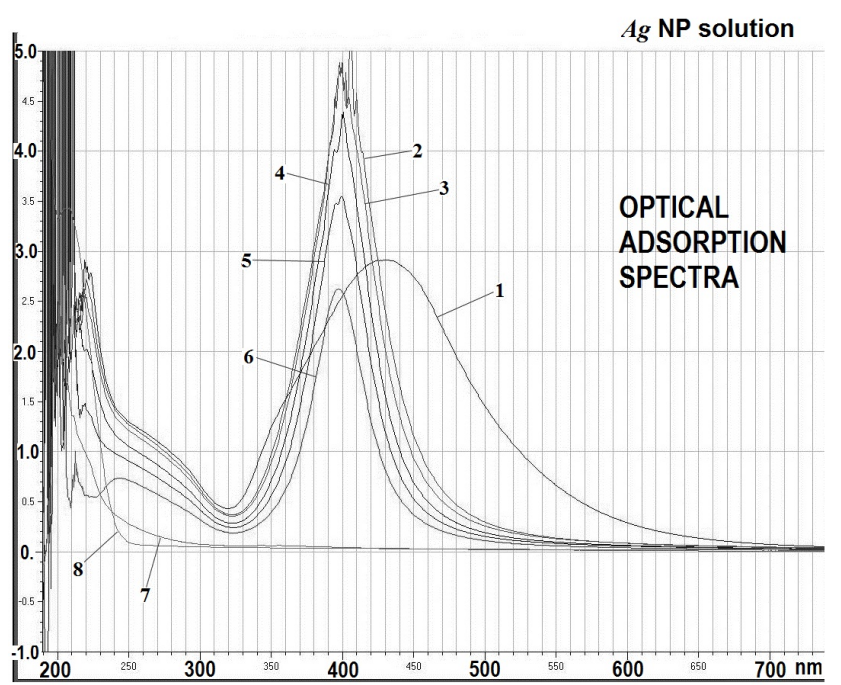

(a) $A g$ NPs

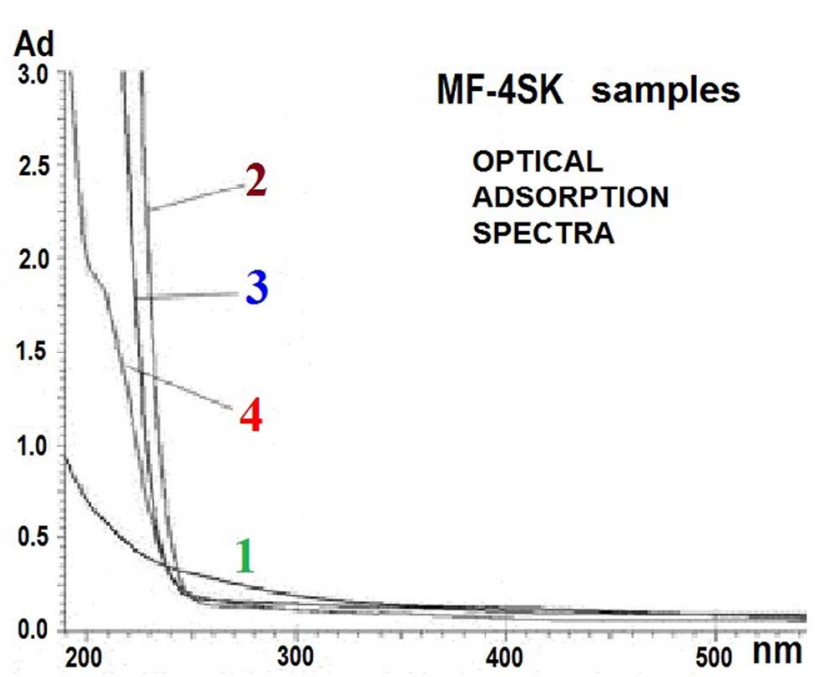

(b) $\mathrm{Ag} \mathrm{NPs}+$ membrane samples

Figure 5. (a) Evolution of OAS (190 - $800 \mathrm{~nm})$ for $\mathrm{Ag}$ NPs $(\omega=8.0)$ solution (without dilution) after contact with MF-4SK membrane: 1-initial moment (0 min), 2-15 min, 3-30 min, 4-90 min, 5-220 min, 6-255 min, 7-24 hours, 8-after 48 hours and (b) OAS (190 - $600 \mathrm{~nm})$ for different MF-4SK membrane samples + RadChem $\mathrm{Ag}$ NPs, $\omega=8.0)$. 1-initial membrane, 2-membrane + NPs (without dilution), 3-membrane + NPs (dilution 1:1), 4-membrane + NPs (dilution 1:2).

The colorless MF-4SK membrane, which was immersed in these solutions becomes coloured, due to the sorption of the $P d$ NPs into/on the membrane. The initial OAS of the colorless membrane is presented on Figure 4(b) (curve 1). The membrane got an adsorption maximum at 280 $\mathrm{nm}$ after 26 hours of the contact with solution (Figure 4(b), curves 3, 4). The evolution of the optical adsorption spectra (OAS, $190-500 \mathrm{~nm}$ ) for the $P d$ NPs solution after contact with the MF-4SK membrane for the various time periods is shown in Figure 4(a). It was found that the metal $P d$ NPs may be introduced into the polymeric matrix MF-4SK practically completely (Figure 4(a), curve 4). Meanwhile the corresponding OAS of the colored membrane samples is depicted in Figure 4(b) (curves 3,4). Comparison of the curves 3 and 4 (Figure 4(b)) shows that the maxima of the adsorption bands for these two curves are proportional to the $\omega=3.0$ (curve 3); 5.0 (curve 4) values.

The optical adsorption spectra for the $A g$ NPs solutions with the solubilization degree $\omega=8.0$ are represented in Figure 5(a).

The initial micellar solutions of $A g$ NPs $(\omega=8.0)$ without dilution (Figure 5(a), curve 1) and with different amounts of dilution (1:1, 1:2 by inert solvent-isooctane; not shown here) have a band with the same wide adsorption maximum at $430 \mathrm{~nm}$. The band with this maximum (curve 1 without dilution) disappears after contact with MF-4SK membrane after 15 minutes. It should be explained by the transformation of the $A g$ NPs in the solution into the NPs of smaller sizes. This is the reason for the peak shift and its subsequent narrowing (Figure 5(a), transfer from peak 1 to the next ones). The newly formed band with the sharp adsorption maximum at $390 \mathrm{~nm}$ (Figure 5(a), curves 2-6) diminishes gradually and completely disappears after 24 hours (curve 7).

It follows from these data that contact the membranes with the NPs micellar solution leads to the fast size decreasing (during $15 \mathrm{~min}$ ) of the $\mathrm{Ag}$ NPs.

In principle some conclusions concerning the sorption kinetic properties of the MF-4SK membrane (Figure 5(a)) may be done. For the short period $A g$ NPs $(430 \mathrm{~nm})$ are transformed into another nanoparticles $(\lambda=390 \mathrm{~nm})$. Then concentrations of these NPs are decreased due to the adsorption without changing of maximum of the OAS.

The comparison of the OAS of the initial MF-4SK membrane (Figure 5(b), curve 1) with the OAS of the different film samples (Figure 5(b), curves 2-4) shows that the intensity of the spectra depends on the nanoparticle content in the solution.

The AFM data (Figures 6 and 7) show the changes of properties of $P d$ NPs which occur during their adsorption on the MF-4SK membranes.

The AFM data for the $P d$ NPs from the initial solution before is shown in Figure 6. The corresponding changes of the nanoparticles in the solution after contact with the membrane are shown in Figure 7.

Figure 7(b) shows the small fragment of the image of Figure 7(a). Figure 7(d) represents the corresponding z-size (relief) recorded along the ticks indicated in Figure 7(b). Figure 7(c) represents the $P d$ NPs size distribution (surface topography) from solution obtained by AFM method.

The comparison of Figures 6 (before adsorption) with Figures 7 (after adsorption) indicates the size increase 


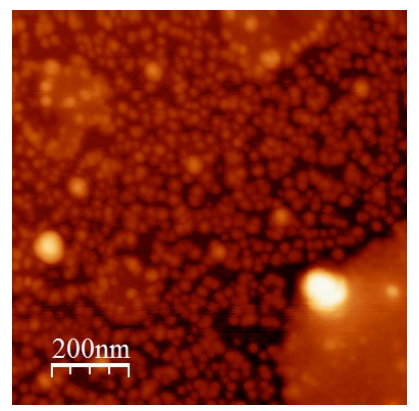

(a)

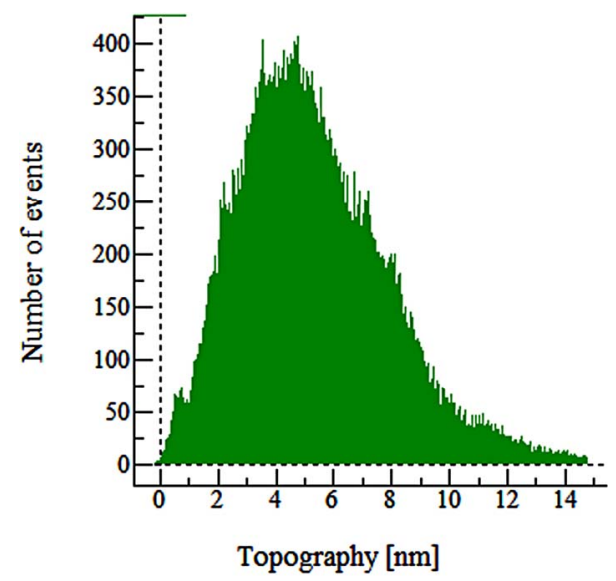

(b)

Figure 6. AFM data for initial $\boldsymbol{P d}$ NPs solution with $\omega=\mathbf{5 . 0}$ before adsorption: (a) AFM image and (b) AFM surface topography of $\boldsymbol{P d}$ NPs from solution.

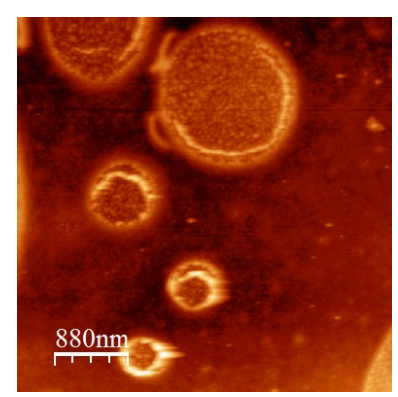

(a)

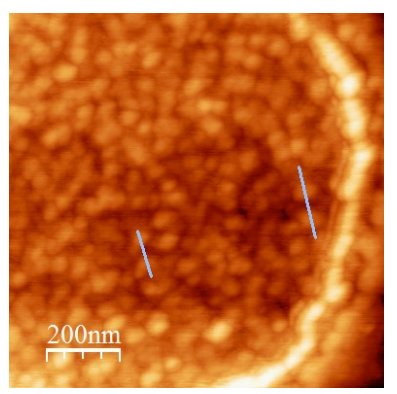

(b)

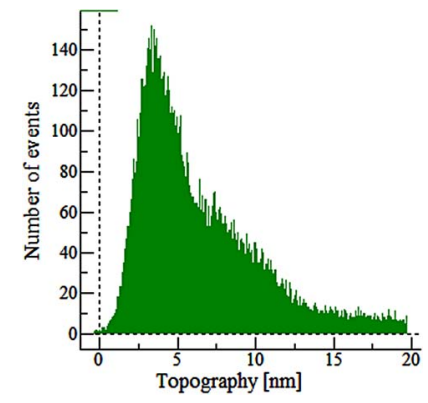

(c) and the agglomeration of the $P d$ NPs after contact of the solution with the membrane surface. This fact may be explained by the deformation of the $P d$ NPs which promotes the $P d$ NPs agglomeration.

The results obtained by using scanning electron microscopy (SEM, Figure 8) indicate surface adsorption and incorporation of the metal $P t$ NPs and $P d$ NPs inside the polymer matrix of the metal-polymer Nf films.

The optical absorption spectra (OAS) of solutions with the solid membrane $\mathrm{Nf}$ in the absence and in the presence of $P t$ (and $P d$ ) NPs are shown in Figure 9(a) (and Figure 9(b)) [10].

The Pt NPs have a pronounced plasmon absorption band in the range $200-400 \mathrm{~nm}$ with maxima at $230-270$ $\mathrm{nm}$. Its intensity increases with the increase of $P t$ content (Figure 9(a)). The content of the Pt NPs depends on the solubilization coefficient value $(\omega)$.

Figure 9(b) shows the OAS for $P d$ NPs: 1 -initial $P d$ solution; 2-in micellar solution; 3 - in Nf film; 4 -initial Nf film. The comparison of curves 2 and 3 leads to the conclusions about the changes in sizes, shape of the $P d$ NPs. It follows that the sizes of $P d$ NPs are smaller in the Nf film than in the primary solution.

The functional activity of the nanocomposites: $P t$ NPs/Nf (a), $P d$ NPs/Nf (b) was confirmed by the results of the electro-cathalytical investigations [10].

Figure 10 shows CVA for the metal-polymer nanocomposites: $P t$ NPs/Nf (a) and $P d$ NPs/Nf (b). It is seen that at $[P t]$-concentration of $\sim 0.02 \mathrm{mg} / \mathrm{sm}^{2}$ (a), the char-

Figure 7. AFM data for $\boldsymbol{P d}$ NPs from solution with $\omega=\mathbf{5 . 0}$ after adsorption.

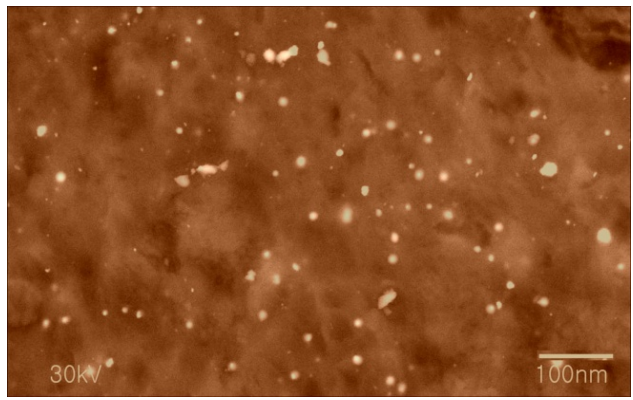

(a)

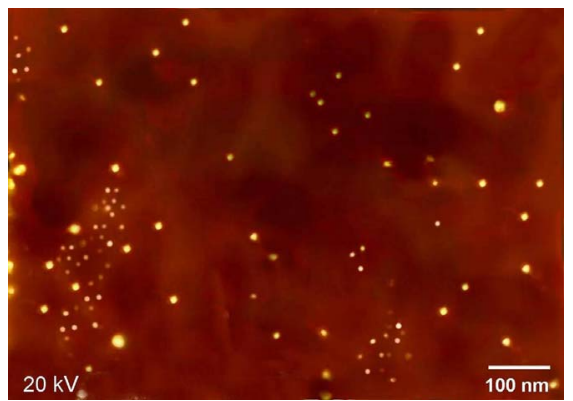

(b)

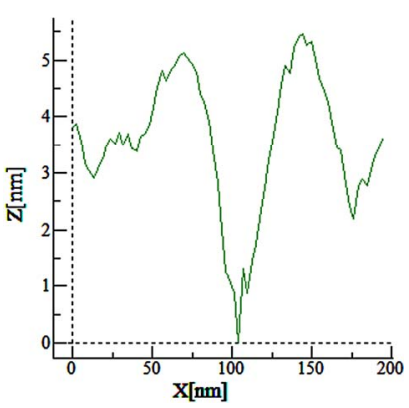

(d)

Figure 8. SEM images of the $P t$ (a) and $P d$ (b) NPs on the surface of the Nf at $\omega=1.5$. 


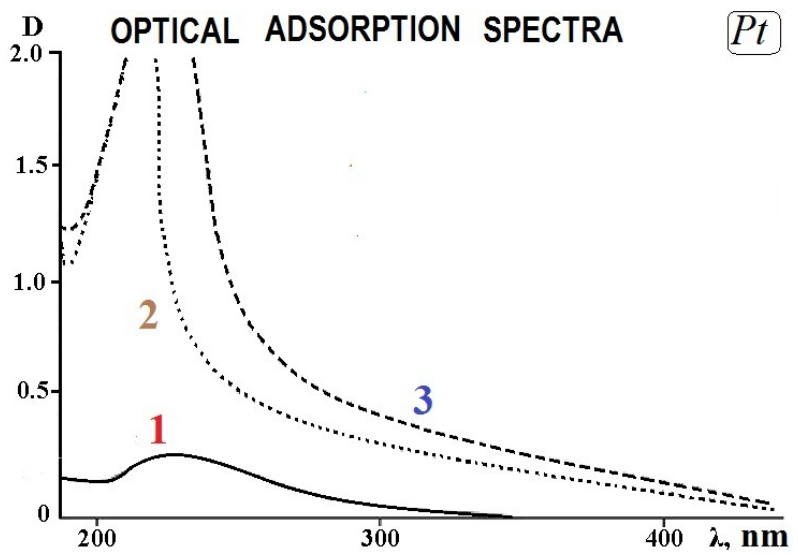

(a)

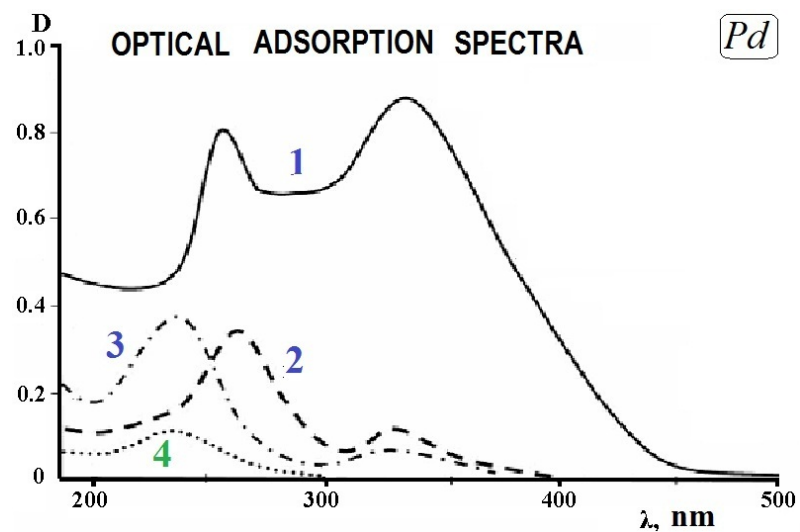

(b)

Figure 9. (a) OAS for Pt NPs. Nf solutions and films with Pt NPs; $1-\mathrm{Nf}$ film without $P t$; $2-\mathrm{Nf}$ with $P t$ NPs at $\omega=1.5$; $3-\mathrm{Nf}$ with $P t$ NPs at $\omega=3.0$ and (b) OAS for $P d$ NPs. Nf solutions and films with: 1 -initial $P d$ solution at $\omega=1.5$; 2 -Nf solution with $P d$ NPs; 3-Nf film with $P d$ NPs; 4 - initial Nf film without $P d$.

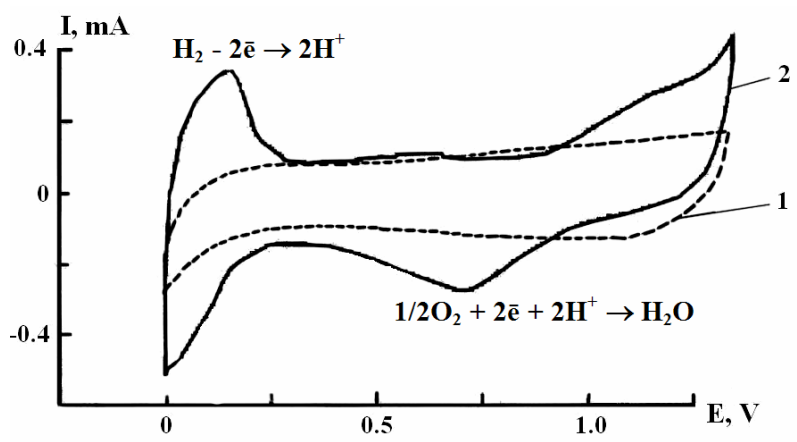

(a)

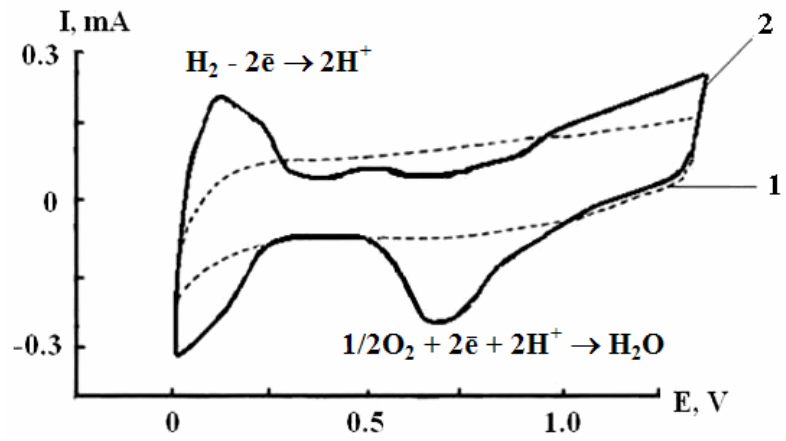

(b)

Figure 10. CVA of metal-polymer nanocomposites: Pt NPs/Nf (a), Pd NPs/Nf (b); (a) Pt, $0.02 \mathrm{mg} / \mathrm{sm}^{2} \mathrm{solutions,} \omega=1.5$ (dashed curve 1); to $P t, 0.19 \mathrm{mg} / \mathrm{sm}^{2}$ with $\omega=5.0$ (solid curve 2) and (b) $P d, 0.04 \mathrm{mg} / \mathrm{sm}^{2}$ solutions, $\omega=1.5$ (dashed curve 1 ) to $P d 0.19 \mathrm{mg} / \mathrm{sm}^{2}$ with $\omega=5.0$ (solid curve 2 ).

acteristic peaks related to adsorption/desorption of hydrogen $(0-0.3 \mathrm{~V})$ and oxygen $(>0.6 \mathrm{~V})$ are poorly expressed (Figure 10). This is probably due to the relatively low $[P t]$-concentration in the investigated samples. At the $[P d]$-concentration of $0.19 \mathrm{mg} / \mathrm{sm}^{2}$ (b) the CVA is typical for $P d$ peaks of the adsorption/desorption of hydrogen and the area of restoration for molecular oxygen $(0.6-0.9 \mathrm{~V})$. The same dependence was shown for $P t / \mathrm{Nf}$ (a), i.e. increase of $[P t]$ NPs concentration leads to the appearance of the characteristic adsorption/desorption oxygen peaks.

\section{Conclusions}

The results of the investigations demonstrate the possibility of modification of the both: Nf polymer film and MF-4SK membranes by various metal NPs: $P t, P d$ and $A g$ NPs obtained on the basis of the original author's methods produced in the reverse micelles as microreactor [7].

It was estimated the changes of the NPs size distribu- tions in the Nf membranes in dependence of the solubilization parameter $(\omega)$ of the NPs-micellar solution system.

It was found by using the cyclic voltamperometry (CVA) method that the obtained metal-polymer nanocomposites $P t \mathrm{NPs} / \mathrm{Nf}$ and $P d \mathrm{NPs} / \mathrm{Nf}$ possess high catalytic activity in the anodic oxidation reaction of hydrogen and the cathodic reaction of oxygen reduction in the membrane-electrode blocks of energy sources.

The $P t$ NPs and $P d$ NPs with the modified MF-4SK polymer film appear to be promising electrolytes for Polymer Electrolyte Fuel Cells.

\section{REFERENCES}

[1] K. A. Mauritz and R. E. Moore, "State of Understanding of Nafion," Chemical Reviews, Vol. 104, No. 10, 2004, pp. 4535-4586. doi: 10.1021/cr0207123

[2] A. K. Sahu, S. Pitchumani, P. Sridhar and A. K. Shukla, "Nafion and Modified-Nafion Membranes for Polymer Electrolyte Fuel Cells: An Overview," Bulletin of Materi- 
als Science, Vol. 32, No. 3, 2009, pp. 285-294. doi:10.1007/s12034-009-0042-8

[3] Y. L. Cao, X. L. Ding, H. C. Li, Z. G. Yi, X. F. Wang, J. J. Zhu and C. X. Kan, "Morphology-Controllable Noble Metal Nanoparticles: Synthesis, Optical Property and Growth Mechanism," Acta Physico-chimica Sinica, Vol. 27, No. 6, 2011, pp. 1273-1286.

[4] Yu. A. Dobrovolskii, A. V. Pisareva, L. S. Leonova and A. I. Karelin, "New Proton-Conducting Membrane for Fuel Cell and Gas Sensors," Alternative Energy and Ecology, Vol. 12, No. 20, 2004, pp. 36-38.

[5] T. K. Jain, M. Varshney and A. Maitra, "Structural Studies of Aerosol OT Reverse Micellar Agregates by FT-IR Spectroscopy," Journal of Physical Chemistry, Vol. 93, No. 21, 1989, pp. 7409-7416. doi:10.1021/j100358a032

[6] A. G. Dokuchaev, T. G. Myasoedova and A. A. Revina, "The Studying of Different Factors Effect on the Silver Aggregation Under $\gamma$-Radiation in the Reverse Micelle,"
Chim. Vysokich Energii, Vol. 31, No. 5, 1997, pp. 353357.

[7] A. A. Revina, "Specimen of the nanostructural Metal Particles and the Method for Its Preparation," RF Patent No 2322327, 2008.

[8] T. K. Jain, M. Varshney and A. Maitra, "Determination of Size Parameters of Water-Aerosol OT-Oil Reverse Micelles from their Nuclear Magnetic Resonance Data," Journal of Physical Chemistry, Vol. 88, No. 21, 1984, pp. 5122-5125. doi:10.1021/j150665a064

[9] D. A. Tanasjuk, V. V. Zeitlin, A. A. Revina and V. I. Ermakov, "Electroconductivity and Electrolysis in Reverse Micelles," 2012.

[10] N. A. Yashtulov, A. N. Bolshakova, A. A. Revina and V. R. Flid, "Metall-Polymer Nanocomposites Based on Platinum Nanoparticles for Chemical Fuel Cells," Russian Chemical Bulletin, Vol. 60, No. 8, 2011, pp. 1-5. doi:10.1007/s11172-011-0235-3 